\title{
Las tecnologías convergentes (nanotecnología, biotecnología y las ciencias cognitivas) y su relación con la bibliotecología
}

\section{Ariel Antonio Morán Reyes}

Artículo científico| Recibido: 14 de enero del 2019| Corregido: 08 de junio del 2019| Aceptado: 14 de junio del 2019| Publicado: 01 de julio del 2019

\section{DOI: 10.15517/eci.v9i2.35897}

e-Ciencias de la Información, volumen 9, número 2, Jul- Dic 2019

ISSN: 1649-4142

\section{다(i)(2)}





\title{
Las tecnologías convergentes (nanotecnología, biotecnología y las ciencias cognitivas) y su relación con la bibliotecología
}

\author{
Converging technologies (nanotechnology, biotechnol- \\ ogy and cognitive sciences) and their relationship with \\ Library and Information Science
}

Ariel Antonio Morán Reyes

\section{RESUMEN}

La designación tecnologías convergentes hace referencia a la confluencia de la iencia computacional con campos tecnocientíficos como la Nanotecnología, la Biotecnología y las Ciencias Cognitivas. El objetivo de este trabajo es señalar y analizar los vínculos que guardan estas tecnologías convergentes con la Bibliotecología -que también recurre al uso de las tecnologías de la información y la comunicación-, ya sea en términos metodológicos, conceptuales o de aplicación. Como principal conclusión se deduce que la Nanotecnología, la Biotecnología y las Ciencias Cognitivas han reconfigurado, en los últimos años, sus propios conceptos de información. Esto ha hecho que profesionales de otras disciplinas, que tienen por objeto de estudio a la información, se detengan a re-pensar su naturaleza, dinámica y utilización.

Palabras Clave: Tecnologías convergentes; Bibliotecología y Ciencias de la Información; Tecnologías de la información y la comunicación.

\section{ABSTRACT}

The denomination convergent technologies refers to the confluence of computational science with techno-scientific fields such as nanotechnology, biotechnology and cognitive sciences. The objective of this work is to point out and analyze the links that these convergent technologies keep with Library Science (which also uses the information and communication technologies), either in methodological, conceptual or application terms. The main conclusion is that both nanotechnology, biotechnology and cognitive sciences have reconfigured their own conceptuation of information in recent years. This has caused professionals from other disciplines, who study information, to stop to rethink its nature, dynamics and use.

Keywords: Converging Technologies; Library and Information Science; Information and Communications Technology. 


\section{Antecedentes Teóricos}

Con el término tecnologías convergentes se alude a la sinergia de lasTecnologías de la Información y de la Comunicación (TIC) con la Nanotecnología, la Biotecnología y las Ciencias Cognitivas. Esta convergencia científica se basa en los conceptos unificados de materia a nanoescala, información y biosistemas en todas las escalas, por lo que su objetivo primario es proporcionar nuevas bases para la creación de conocimiento, la innovación y la integración de tecnología en relación con las necesidades humanas. Tanto la Nanotecnología, la Biotecnología y las Ciencias Cognitivas son campos tecnocientíficos, en el sentido que puntualizó Linares (2008)

Uno de los fenómenos sintomáticos del mundo tecnológico es la estrecha co-operación entre la ciencia y la tecnología en un nuevo sistema de acciones pragmáticas que se denomina ahora tecnociencia [...] La tecnociencia vincula la información y el conocimiento científicos, las habilidades y destrezas técnicas para la producción industrial de artefactos y dispositivos tecnológicos [...] Las tecnologías se transforman en tecnociencias en la medida en que se hacen más complejas y requieren el concurso de otras ramas científicas y técnicas, y en la medida en que crean nuevas entidades artefactuales (pp. 367 - 370).

Las tecnociencias tienen claros fines pragmáticos lo que deriva en la apropiación y en la subordinación de investigación teórica, fundiendo las operaciones cognoscitivas y la producción de artefactos en una unidad de acción; pero sus productos principales son modelos pragmáticos y diseños artefactuales, con fines económicos o militares (Echeverría, 2003). Como bien señala Venteño (2013)

La nanociencia, la nanotecnología, las TIC's y la biotecnología se encuentran en una etapa temprana de investigación y desarrollo, en la que el presupuesto de las universidades en este campo se destina a las investigaciones, y las inversiones del sector productivo están dirigidas hacia la investigación y comprensión de los fenómenos de la nanoescala [Sic] (p. 13).

El término tecnologías convergentes comenzó a cobrar fuerza gracias a un reporte técnico-científico, elaborado en junio de 2002, intitulado: Converging technologies for improving human performance: Nanotechnology, biotechnology, information technology and cognitive science, coordinado por el ingeniero en materiales Mihail C. Roco y el sociólogo William S. Bainbridge, ambos adscritos a la Fundación Nacional de Ciencia de los Estados Unidos de América. En este reporte -auspiciado en parte por el Departamento del Comercio- se hace referencia a las tecnologías convergentes, a partir de las siglas NBIC (Nano, Bio, Info y Cogno), cuya "convergencia de diversas tecnologías se basa en la unidad material a escala nanométrica y en la integración de la tecnología desde esa escala" (Roco y Bainbridge, 2003, p. $\mathrm{x})$. Cabe mencionar que dicho documento fue resultado de un taller llevado a cabo en diciembre de 2001, a raíz de varias investigaciones exploratorias en el marco de la presentación de la Iniciativa Nacional de Nanotecnología, proyecto conjunto de la Casa Blanca con una decena de agencias 
gubernamentales y con representantes de varios sectores empresariales interesados en la Nanotecnología. En este reporte se abrió el horizonte para hacer converger a la informática con la Nanociencia, la Biomedicina, la Ingeniería genética y las Neurociencias, ramas que desde algunos años atrás ya comenzaban a desarrollar proyectos de manera paralela.

Las tecnologías convergentes constituyen, entonces, el advenimiento de una nueva generación de tecnologías que han ido transformando radicalmente la producción económica mundial en connivencia con los flujos de información transfronterizos, al grado de considerárseles como la próxima gran revolución industrial, de la cual comenzamos a advertir claros atisbos. No obstante, su simiente y alcances son más complejos. Cada una de las tecnologías que ahora concurren han tenido desde tiempo atrás algunas aplicaciones en una diversidad de campos de la producción, pero su constante nivel de especialización sigue implicando una barrera tanto para su entendimiento y estudio como para la difusión de los mecanismos que las hacen posibles (Pedreño, 2009).

De hecho, en diferentes latitudes, se han suscitado debates sobre la comprensión pública de la ciencia y el carácter participativo de la tecnología en la vida de las personas. Es por ello que, con este artículo, se pretende aclarar la conceptuación de las tecnologías convergentes y puntualizar la influencia que pueden tener en el ejercicio de la Bibliotecología, una disciplina que recurre cada vez más a las TIC. Si bien los campos científicos realizan valiosas aportaciones a la Bibliotecología, también esta les aporta insumos, ya que las tecnologías convergentes requieren de información científica para cumplir muchos de sus cometidos (Sindelar, 2019, p. 241). Por ejemplo, la Biotecnología requiere de diversos sistemas y servicios de información científica y tecnológica, pero, a su vez, produce información y técnicas para su recuperación y gestión. Uno de los productos biotecnológicos más rentables es el procesamiento de bioinformación.

La convergencia entre estos campos se abrió a partir del carácter interdisciplinario de las Ciencias Cognitivas, las cuales crearon un puente con las TIC, a través de la Inteligencia Artificial. Luciano Floridi (2011) argumentó que la Inteligencia Artificial actuó como un "caballo de Troya" que trajo un paradigma computacional/informacional mucho más vasto, revestido con sus propios temas, métodos y modelos, y con su particular punto de vista en conceptos como mente, conciencia, experiencia, conocimiento, verdad. Poco después de que se diversificaran las tecnologías digitales -y las áreas que le dan sustento gracias a diversas aplicaciones-, se dio un enlace entre estas y la computación molecular. En este sentido, la Biotecnología (campo derivado de la genética y la biología molecular) representó una nueva forma de comprensión de la estructura física de lo vivo. Finalmente, la Nanotecnología se acercó a las otras tres a través de intereses comunes derivados las nanociencias y estudios a escalas cuánticas.

Por supuesto, la informática y los estudios aplicados de la tecnología son las que guardan una relación mucho más estrecha con la Bibliotecología, por ello, en este trabajo no se les tomará en cuenta de manera particular -sólo referencial-, y se abocará a la relación de la Bibliotecología -ya sea en términos metodológicos, teóricos o de aplicación- con las Ciencias Cognitivas, la Biotecnología y la Nanotecnología -las cuales también recurren a las TIC de forma constante-. 
Antes de indagar en cuestiones prácticas y de aplicación -donde se despliega el uso de los dispositivos tecnológicos-, es necesario sostener que una de las aportaciones primordiales -mas no la única- de las tecnologías convergentes hacia la Bibliotecología es de tipo conceptual, sobre todo, en lo que respecta a la delimitación de lo que se entiende por información. La principal razón de que su contribución primaria sea sobre procesos de conceptuación estriba, entre otras cosas, en que muchos de los modelos de la Nanotecnología o la Biotecnología surgen a partir del análisis teórico, además de que su idea de información se reconfigura con mayor celeridad, a diferencia de otros campos. A esto habrá que sumar que las Ciencias Cognitivas nutren su reflexión, en gran medida, de los conceptos emanados de la dos anteriores (Gordijn, 2008).

A continuación, se expondrán los vínculos teóricos y los casos de aplicación e implementación práctica que existen entre cada una de las tecnologías convergentes en relación con la Bibliotecología.

\section{La Nanotecnología y la Bibliotecología}

La Nanotecnología, en palabras de Dalton-Brown (2015) es "la ingeniería de sistemas funcionales a nivel molecular [...] La etiqueta 'nanotecnología' parece ser el término más relevante (así como el más ampliamente utilizado) para hablar sobre las tecnologías nanofabricadas que afectan nuestras vidas" (pp. 11-12). Este campo se acercó a los estudios de la información a partir de las bases sentadas por la cibernética, la cual se enfocó en las investigaciones científicas de los mecanismos de control automático y de los problemas de comunicación, tanto en máquinas como en organismos vivos. La Nanotecnología no es únicamente una ingeniería de materiales, sino que se le considera una confluencia de varias áreas de conocimiento -Biología molecular, Mecánica cuántica, Química de materiales, Nanometría, entre otras-. La Nanotecnología se erigió como la modificación posible, a nivel atómico, de prácticamente cualquier material, orgánico o inerte, y esto supuso un alto dominio de la física de partículas y la química cuántica.

Holger Lyre (1996), desarrolló una teoría cuántica de la información, en la que concibió teóricamente un bit cuántico de información potencial -un "ur", que es la unidad física de la teoría-ur-. Esta idea de unidades de información supone una diferencia entre la información sintáctica y la información semántica. La primera se refiere a una cantidad de distinguibilidad estructural, lo que implica que una magnitud se puede medir en bits; la segunda se refiere a la información que existe en virtud de un concepto o en un nivel de significado determinado. Lyre adujo que:

Por ejemplo, una letra impresa en un papel se refiere a diferentes cantidades de información si se considera bajo el concepto de "letra de un alfabeto de una lengua determinada" o bajo el concepto "moléculas de tinta de la impresora" (1996, p. 2268).

Esta diferencia puede ser entendida con los conceptos información cinética e información estructural (Stonier, 1996). Si pensamos en la información que puede contener una unidad USB, sería normal hacer referencia a la información digital que almacena -información cinética-, pero también en la información que se puede obtener del disco material de polietileno que la constituye -composición química, forma, tamaño, masa. 
Ahora bien, la propuesta de Lyre es un modelo de física teórica y, por lo tanto, su aportación puede llegar a ser netamente conceptual. Sin embargo, incluso las teorías cuánticas más clásicas tienen una aplicación directa en la Bibliotecología, específicamente en procesos de recuperación de información. Este conjunto de teorías intenta responder a la interrogante de si todo lo ocurrido en el universo se almacena como información, dentro de un estado de un sistema cuántico. La unidad básica de la información cuántica es el qubit -una medida para la cantidad de información- y a diferencia de los estados digitales clásicos, un sistema de dos estados cuánticos puede darse en una superposición de los dos estados en cualquier momento dado. Mediante el estudio de la relación entre la teoría cuántica y la información, es posible diseñar nuevos tipos de máquinas, por ejemplo, una computadora cuántica, con mucho mayor alcance que lo avizorado por los estudios de la Inteligencia Artificial. Su diseño apelaría a la física digital, o sea, que recurre a la premisa de que el universo puede ser descrito a través de la información y por aquello que puede ser computable.

Por supuesto, a pesar de que esto tendría en el papel muchas implicaciones en el área de la recuperación de la información, su implementación resulta, hasta la fecha, demasiado onerosa -incluso para las universidades más connotadas-. Los pocos proyectos que pueden utilizar estos procedimientos son de carácter interinstitucional -entre universidades, agencias del gobierno y empresas privadas-. El uso más prometedor para un dispositivo de búsqueda cuántica es la factorización cuántica. La búsqueda inteligente de la información permitiría a los usuarios de bases de datos documentales realizar un rastreo textual en sistemas de información utilizando frases y contexto, con lo que se reducen las deficiencias de los buscadores convencionales, pues se omiten los múltiples resultados carentes de sentido a los requerimientos de información. Un modelo basado en un algoritmo de búsqueda cuántico podría elevar las potencialidades de una hoja electrónica para construir sistemas de información y/o sistemas inteligentes. La información se reorganizaría para que pueda ser representada en forma sincrónica, en forma diacrónica o como texto.

En general, la perspectiva de bits cuánticos busca establecer el estado de un sistema con precisión, bajo el cual el contenido de información de un mensaje puede medirse en términos de la cantidad mínima para almacenar el mensaje en un sistema de doble nivel cuántico. Un sistema de doble nivel cuántico puede llevar un máximo de información, en el mismo sentido que un dígito binario clásico puede llevar a lo sumo un bit clásico (Galindo, 2008). Se espera que esta clase de tecnologías cobre un carácter más participativo e inclusivo y comience a introducirse, paulatinamente, en áreas de la vida cotidiana, como las universidades y bibliotecas académicas.

Aunque la factorización cuántica parece prometedora tiene sus límites, como los tiene cualquier herramienta tecnológica, además de la dificultad cognoscitiva que supone el manejo de un dispositivo semejante. Un procedimiento de este tipo es parte de lo que Andrew Shenton (2009) denominó como búsqueda de información inferencial, la cual

[...] abarca situaciones en las que las personas siguen pasos para encontrar información a través de métodos tortuosos que entregan material que puede estar ampliamente relacionado con la necesidad pero que puede no ser inmediatamente utilizable, y que al tener 
acceso a lo que reciben, gasten un considerable esfuerzo cognitivo en su aplicación directa a sus propias situaciones (p. 353).

Este autor ha comentado que hay necesidades de información imposibles de satisfacer, por ejemplo, "es imposible dar una respuesta precisa a la pregunta: ¿Cuántas estrellas hay en la galaxia?" (Shenton, 2009, p. 357). La búsqueda de información inferencial es, por definición, una acción indirecta, pues la búsqueda no se basa en puntos de acceso directos, al menos no en una instancia inmediata, sino en una búsqueda indeterminada en volúmenes inmensos de información -como los big data-, el mismo autor menciona, además, que la dimensión de la búsqueda de información inferencial varía enormemente de una situación a otra, por diversos factores, cuantimás tratándose de factorización cuántica. A pesar de que

La mecánica cuántica no proporciona una predicción tan reconfortante, pero hace un trabajo mucho mejor [...] El mundo clásico y el mundo cuántico parecen estar muy separados. Sin embargo, a medida que avanzamos a lo largo de la escala, de la más grande a la más pequeña, las reglas clásicas eventualmente dan paso a las reglas cuánticas. El medio oscuro y turbio entre los dos dominios es la provincia de la nanotecnología [...] el ciber-software está entrando en el pasado, porque las nanotecnologías realmente ofrecen increíbles beneficios. Gracias a una nueva tecnología nanoquirúrgica que abre la posibilidad de que una mano protésica se vea, se mueva y se sienta como la real, tenga un sentido del tacto y esté controlada por el cerebro. Los implantes de células cerebrales, pequeños e invisibles, pueden mejorar la memoria, cambiar el estado de ánimo y controlar brazos, piernas y otras partes artificiales; pueden almacenar información igual a varias bibliotecas grandes (Shunin, Bellucci, Gruodis y Lobanova-Shunina, 2018, pp. 352-353).

Pese a la complejidad de una búsqueda de información cuántica, Zibareva (2015), considera que muchos de los motores de búsqueda convencionales no cubren los alcances que aquellas herramientas que paulatinamente incorporan métodos de la computación cuántica, los cuales proporcionan

[...] el alcance sustantivo y temporal de los datos, y la frecuencia de su actualización; la naturaleza de la indexación; capacidades de búsqueda; modos de visualización, consulta, carga y análisis de resultados; así como facilidades de información y soporte para el usuario [...]. Desde la perspectiva de la información, el problema clave consiste en lograr la cobertura más amplia posible (idealmente completa) de la matriz de documentos correspondiente. El uso individual de las bases de datos multidisciplinarios y especializados y los sistemas de recuperación de datos no aseguran dicha cobertura debido al conjunto limitado de fuentes de referencia (pp. 101-102).

Entonces, las aplicaciones más claras de la Nanotecnología hacia la Bibliotecología pueden advertirse en algunos modelos teóricos para la recuperación de información. No obstante, gracias a la química de materiales han existido, también, aportaciones en los procesos de preservación y conservación. Dichos procesos no son tan recientes como pudiera pensarse, como algunos medios de almacenamiento, por ejemplo, los discos magnéticos, los cuales, al ser leídos poseen una capacidad que 
[...] oscila alrededor de treinta y dos Gigabytes por pulgada cuadrada - en un disco que además sólo pesa cien gramos y ocupa menos de cien centímetros cúbicos - en los discos magnéticos más recientes. El almacenamiento magnético actual cae ya totalmente en el campo de la nanotecnología, es decir, partículas menores a 100 nm (Voutssás, 2011, p. 307).

Si bien lo anterior es cierto, el gran impacto de las nanotecnologías en la gestión de la información surgió con la cuarta generación de las computadoras, en los años setenta del siglo pasado, cuando las unidades de memoria con núcleo magnético comenzaron a ser reemplazadas por aquellas de chips de silicio. El chip fue resultado del proceso de microminiaturización de la nanociencia, el cual comprime los elementos de circuitos en un espacio cada vez más pequeño.

\section{La Biotecnología y la Bibliotecología}

De acuerdo con Recasens (2018), "La biotecnología moderna se basa en la ingeniería genética, entendida como la manipulación genética de organismos vivos a fin de que produzcan productos útiles [...] si la biotecnología debe ser efectiva, ha de generar productos en cantidades apreciables" (p. 11). Como ya se mencionó, "no es ningún secreto que la biotecnología está ligada a la tecnociencia corporativa y busca sobre todo generar productos rentables. De hecho, su aplicabilidad comercial es una de sus características definitorias" (Weisberg, 2015, p. 41). A pesar de esto, muchas de las aplicaciones de las tecnologías convergentes en los procesos de transmisión de la información tienen que ver con las exigencias sociales sobre las aplicaciones tecnocientíficas, que propugnan para que estas cobren un carácter menos comercial y mucho más participativo en las vidas de las personas y de las sociedades.

En el apartado anterior, ya se estableció que la Nanotecnología supone un alto conocimiento de física de partículas para la factorización cuántica, lo cual también involucra a la Biotecnología. Muchos modelos teóricos de factorización en escalas nano han sido aplicados en la recuperación y gestión de la información. Chen, Qiang y Zhang (2014), encontraron que un análisis teórico que realizaron sobre tecnologías de hibridación para el procesamiento de información del ADN, resulta también "útil para la búsqueda y transmisión paralela de información, desde diferentes fuentes específicas hacia diferentes destinos, lo que ofrece una tecnología realista para la flexibilidad de la gestión de la información" (p. 29).

Ahora bien, la relación de la Biotecnología con los estudios de la información se gestó desde un inicio de manera muy similar a la Nanotecnología, ya que se dio con la influencia de la cibernética de Norbert Wiener -en colaboración con el mexicano Arturo Rosenblueth-, y sus trabajos sobre la transmisión de información entre el músculo cardiaco y las ondas neuronales del cerebro -la misma formulación matemática puede aplicarse en ataques de migraña o una arritmia cardíaca- (Wiener y Rosenblueth, 1946). Esta relación se clarifica aún más con la denominada cibernética de segundo orden y conceptos como homeostasis o comportamiento sin propósito (Rosenblueth, Wiener y Bigelow, 1943), que designa a un conjunto de procesos de auto-organización en organismos vivos, pero, también, en un entorno social, para mantener el equilibrio, a partir de la disposición de medios para la adquisición, uso, retención y transmisión de información. 
Las necesidades y la complejidad de la vida moderna plantean a este fenómeno del intercambio de informaciones demandas más intensas que en cualquier otra época; la prensa, los museos, los laboratorios científicos, las universidades, las bibliotecas y los libros de texto han de satisfacerlas o fracasarán en sus propósitos [...] El proceso de recibir y utilizar informaciones consiste en ajustarnos a las contingencias de nuestro medio y de vivir de forma efectiva dentro de él [...] Vivir de manera efectiva significa poseer la información adecuada (Wiener, 1988, pp. 17-18).

Esta nueva manera de entender la realidad mostró que la podemos encontrar información en el conjunto de letras del alfabeto latino o en los nucleótidos de un fragmento de ADN

[...] para el "ingeniero de conocimiento" que trata de desarrollar una teoría general de la información esta consideración parece plausible e interesante. En lugar de contemplar una disposición ordenada de partículas físicas organizadas formando un cristal, consideremos una disposición de símbolos humanos organizados en una estructura simbólica determinada (letras del alfabeto latino más signos de puntuación, organizadas formando una frase) [...] Moléculas tales como el ADN y las proteínas consisten en secuencias o hileras de moléculas más simples repetidas (nucleótidos, aminoácidos). Tales secuencias representan una serie de mensajes. Esos mensajes (las moléculas que los materializan) puestos dentro del contexto de una célula pueden tener"sentido" para la célula [...] Los sistemas biológicos aparecen, de alguna manera, ocupando un lugar intermedio entre los cristales inorgánicos a $0^{\circ} \mathrm{K}$ y los sistemas simbólicos humanos (Stonier, 1996, pp. 77 y 81-82).

La Biotecnología se diversifica en campos como la Robótica, la Zootecnia, la Ingeniería genética y, por supuesto, a la Bioinformática y la Genómica. Es por ello que la relación entre la Biotecnología y la Bibliotecología no necesariamente debe ser directa por sus contenidos; por el contrario, puede ser de índole teórica o metodológica. Por ejemplo, la Bioinformática ha aportado nuevas formas de entender el constructo información y, por lo tanto, se tiende otro vínculo, a su vez, con las Ciencias Cognitivas, en cuanto a que esto se relaciona con la manera en que estructuramos la información en nuestra mente, y de ahí con lo que se entiende por procesamiento de información, ya sea en humanos o en máquinas

La bioinformática está un poco en desventaja en comparación con la informática de los físicos o matemáticos. Los científicos de estas áreas suelen tener una idea de lo que están trabajando y a menudo saben que el mensaje en cuestión (incluso codificado) es significativo, es decir, se trata verdaderamente de información [...] El genoma humano contiene alrededor de 60 gigabits de 'información' (sin comprimir). Razón por la que suponemos que se trata de información biológica importante, ya que ha permitido que las especies existan por lo menos 35,000 años y que ha sido cuidadosamente preservada de generación en generación (Biro, 2011, p. 138).

Años antes, Carl von Weizsäcker indicó que las estructuras biológicas o, en términos más generales, la evolución misma, pueden ser concebidas como "un crecimiento de formas medido como un crecimiento de información" 
(1949, p. 173). Dichas formas pueden considerarse como potencialmente cognoscibles, con lo cual pasamos de una noción objetiva de la información a una subjetiva. Además, un organismo puede entenderse en su totalidad, según este autor, como "semántica objetivada". A partir de lo anterior, podemos mencionar que existen tres tipos de información, a saber:

- La información como realidad (también conocida como información ambiental): esta perspectiva no se interesa en la utilidad, relevancia, significado, interpretación o pertinencia de la información, sino en el nivel de detalle, disposición, localización y frecuencia de los datos no interpretados.

- La información acerca de la realidad (o información semántica): definida así ya que se piensa en una proposición verdadera, es decir, si y sólo si el estado de cosas que expresa se da en la realidad. Este tipo de información reduce el grado de incertidumbre, a partir de un determinado estado de conocimiento del individuo informado. La información aquí se localiza en las estructuras profundas de los documentos y es esencialmente contextual. La información sobre la realidad puede tener enfoques probabilísticos, modales, sistémicos, inferenciales o significativos, pero todos buscan exponer el contenido semántico mediante la adopción de una orientación proposicional.

- La información de la realidad: se advoca al gran complejo de asistentes para ayudas e interacciones: instrucciones, la información genética, algoritmos, órdenes o recetas (Floridi, 2011, p. 30).

Bajo esta tónica, la información sería comprendida, según la Biotecnología y la Nanotecnología, como "información acerca de la realidad"; la información desde la perspectiva de la informática sería vista como "información de realidad", y la información desde el punto de vista de las ciencias cognitivas sería conceptuada como "información acerca realidad".

\section{Las Ciencias Cognitivas y la Bibliotecología}

En las tres conceptuaciones anteriores sobre la naturaleza de la información, ofrecidas porFloridi (2011)-la información comorealidad, la información acerca de la realidad y la información de la realidad-, se considera principalmente a la información que está fuera de la mente -en el mundo fenoménico-, pero las Ciencias Cognitivas consideran, principalmente, a la información que se encuentra instalada en la mente -en términos evolutivos- $y$ también a aquella que puede ser, abstraída, creada y reproducida por la mente. Por ejemplo, en prácticas socio-culturales como la lectura se tiene conceptuado un mundo de la información en el cual pululan diferentes objetos de información-desde una piedra hasta un libro- susceptibles o no de ser comprendidos por el sujeto. La información de esos objetos podría ser información como realidad o información de la realidad -o, en pocas palabras, información fuera de la mente-. Las Ciencias Cognitivas investigan, precisamente, la posibilidad de comprensión de este tipo de información. En concreto, las llamadas Ciencias Cognitivas son

[...] una colección de disciplinas que estudian la mente como un proceso funcionalmente explicable, inspirado en el procesamiento de la información. Por supuesto, esto excluirá del dominio de las ciencias cognitivas algunas de las teorías que afirman que la mente no puede ser "funcional" por naturaleza, o que la mente no tiene 
nada que ver con el procesamiento de la información; pero sí, estos puntos de vista deberían excluirse, ya que tales afirmaciones están intrínsecamente en desacuerdo con los fundamentos de la ciencias cognitivas (Bellini-Leite, 2017, p. 176).

El realismo científico hace parte de tales teorías, ya que suele considerar con mayor preponderancia a la información fuera de la mente, ya que asegura que la información existe, independientemente de que la mente la capte o la interprete

[...] la "información" no constituye un mero producto de la mente humana (una construcción mental para ayudarnos a entender el mundo en que habitamos) sino que se trata de una propiedad del universo: La información es tan real como la materia y la energía (Stonier, 1996, p. 127).

A partir de esto, según la perspectiva cognoscitiva, la información y el significado no son lo mismo: "La información proporcionada por un libro es función del ambiente intelectual preexistente en formas de estructuras de conocimiento en el seno del cerebro del lector" (Stonier, 1996, p. 38). En otras palabras, nuestra percepción mental no determina si algo contiene menos o más información: la información está ahí. El hecho de que no podamos comprender un texto en otro idioma no significa que no haya información, sino, más bien, que nuestra mente no puede interpretarla. Aunque el "lector" no entienda completa o parcialmente un mensaje, cualquiera puede reconocer las letras, y el libro aún mantiene sentido en dos niveles: el libro como un objeto, y las letras como signos. El lector no entiende el mensaje, pero sabe que las letras representan un mensaje; por ejemplo, que las letras pertenecen y estructuran un código.

Además de la información como realidad o la información de la realidad, las Ciencias Cognitivas consideran, también, a la información acerca de la realidad -información creada/reproducida/validada por la estructura mental del sujeto-. León Olivé reconoció como información a aquella que procede de la naturaleza y que está fuera de la mente, pero la consideró como información acerca de la realidad no como información como realidad o información de la realidad. En otras palabras, para él no hay información sin un agente que la valore o la interprete. Para este autor

La información está constituida por datos que representan estados del mundo. La información se acumula, se transmite y puede utilizarse. Y si bien no existen 'datos en sí mismos' ni 'información en sí misma', sino los datos - y la información en general- siempre lo son al menos para un potencial intérprete y usuario, la información se vuelve valiosa sólo cuando intervienen agentes intencionales que valoran esa información y la incorporan a su acervo de conocimiento, con lo cual se afectan tanto su visión del mundo como sus capacidades para la acción y en especial para la transformación del entorno (Olivé, 2007, p. 49).

En esta conceptuación no se intenta dar primacía solo a la perspectiva de las Ciencias Cognitivas. Por el contrario, se da a entender que esta se alimenta de las perspectivas que estudian al mundo en sus diferentes dimensiones, incluidas las de la Nanotecnología y de la Biotecnología, las cuales representan enfoques de información primordialmente tangible. Algunas propuestas, como la teoría de la información representacional, intentan calcular la 
complejidad estructural de la información disponible antes y después de que se ejecute un proceso cognoscitivo. Conceptos como "poder cognitivo, eficiencia cognitiva, densidad cognitiva y el factor de aumento pueden ser usados para estudiar esta nueva era de interacción humano/computadora" (Fulbright, 2017, p. 52).

Patricia Hernández (2009), precisamente, ha propuesto métodos propios desde la mirada de las Ciencias Cognitivas, con el objetivo de entender a las personas usuarias como agentes que hacen parte de un fenómeno complejo. Algunos de estos son cercanos a la Psicología cognitiva, que es la base del entendimiento de los procesos mentales de los sujetos. Según lo dicho por esta investigadora, cada persona usuaria se convierte en el eje alrededor del cual giran las parcelas del conocimiento bibliotecológico. Para consumar el modelo de las Ciencias Cognitivas, esta autora contempla el cálculo computacional, y la perspectiva interdisciplinaria -Filosofía, Psicología cognitiva, Inteligencia Artificial, Neurociencia, Lingüística, Antropología social- (Hernández, 2013). Es indudable que

El aumento de la capacidad tecnológica abre las puertas a nuevas perspectivas desde las ciencias cognitivas y la psicología acerca del uso intensivo de datos (es decir, investigaciones que aprovechan el uso de datos complejos o de gran escala para comprender la cognición y el comportamiento humanos) (Paxton y Tullett, 2019, p. 47).

En consonancia, Amanda Spink y Charles Cole (2004), también se acercaron al estudio de la estructura cognoscitiva del usuario para comprender su comportamiento informativo-específicamente, desde la Psicología evolutiva. Ambos conceptúan a las necesidades de información como requerimientos primarios asociados a un estado en que la información actúa como un recubrimiento del exterior -en el Psicoanálisis se trataría del yo, como una instancia protectora del ello, que es la realidad-. La información, entonces, está encaminada a la adaptación y a la supervivencia, y el comportamiento informativo sería un proceso mental de cada ser humano, basado en el desarrollo modular de su arquitectura cognitiva. La comprensión de la persona usuaria -tanto de su comportamiento y de su arquitectura cognitivapuede ser utilizada para realizar de una manera mucho más eficiente la satisfacción de las necesidades de información.

Autores como García (1995) o Guzmán (2005) proponen tomar en cuenta a cada persona usuaria de una manera predominante, lo que conlleva reconocer sus diferentes dimensiones y complejidades -valiéndose de la Lingüística o la Psicología-. De hecho, García (2011) ha modelado el procesamiento humano de la información y la arquitectura del sistema cognitivo humano, a través de tres estratos: la percepción -sensaciones y recepción de datos-, el razonamiento -procesamiento y uso de la información y el comportamiento informativo- y la memoria -retención a largo plazo y el olvido-. La bibliotecóloga mexicana, Guadalupe Vega (2009), realizó in extenso una investigación sobre la alfabetización informativa desde el enfoque de las Ciencias Cognitivas, en la cual se ahonda en el procesamiento de la información en estudiantes de educación básica. Por su parte, Silva y Lima (2011) señalan un vínculo primario entre las Ciencias Cognitivas y la Bibliotecología, relacionado con la capacidad humana para el procesamiento de la información, específicamente: la relación entre el proceso cognitivo de comprensión de las características de los objetos de información -como unidades de conjunto- y su organización -catalogación, clasificación e 
indización-. Estos autores proponen utilizar a la Ciencia Cognitiva para reformular y contrastar los principales conceptos de la Bibliotecología, por ejemplo, el de "clasificación", a la luz de una comprensión socio-cognitiva, Lingüística, o de interacción con el medio.

En una investigación, que está por publicarse este año en la Journal of Librarianship and Information Science, se sintetiza que la principal aplicación de las Ciencias Cognitivas para las Ciencias de la Información está vinculada

[...] a la investigación sobre el comportamiento y uso de la búsqueda de información [...] esta teoría ha sido valiosa en investigaciones sobre motivaciones para buscar información, compartir conocimientos y aprender. El tratamiento de la teoría cognitiva social en la literatura de la ciencia de la información como pertinente a dos temas se explica a continuación: (1) búsqueda, comportamiento y uso de la información (incluida la alfabetización informativa) y (2) el intercambio de conocimientos (Middleton, Hall y Raeside, 2019, p. 4).

No son pocas las personas que investigan el tema, quienes consideran que la principal aplicación de las Ciencias cognitivas a la Bibliotecología se da con la realización de estudios de comportamientos asociados con el uso de la información. Por ejemplo, estudios de la evaluación de la alfabetización informativa y los niveles cognoscitivos (Dolničar, Boh-Podgornik, Sajovic, Šorgo y Bartol, 2016), o análisis conceptuales de las barreras cognitivas -tanto del bibliotecario como de los usuarios-y su impacto en la búsqueda de información. De hecho, en un estudio del eminente profesor Reijo Savolainen (2015) se determinó una tipología de seis barreras cognitivas:

- La incapacidad para percibir las necesidades de una persona como necesidades de información.

- La incapacidad para articular sus necesidades de información.

- El desconocimiento de las fuentes de información.

- La baja autoeficacia.

- La poca capacidad de búsqueda e incapacidad para manejar la información.

- La sobrecarga.

Algunos autores consideran que estas disfunciones cognoscitivas recaen más en la figura de la persona profesional en Bibliotecología que en la de las personas usuarias

La biblioteca como institución social comenzó a mostrar signos claros de disfunción. Las necesidades sociales y de información alteradas, es decir, el conocimiento y la información analítica en lugar de los documentos, dejaron de incorporarse adecuadamente en la estructura y funciones de las bibliotecas. Esto indica que la biblioteca moderna no cumple con su propósito cognitivo y de información, no hay una integración observada de enfoques cognitivos y comunicativos a las actividades de la biblioteca, que es la razón por la que las opiniones con respecto a la necesidad de la amalgama de todas las bibliotecas, el riesgo de abandonar el libro como un medio de información que requiere trabajo individual para el procesamiento de esta información, cambiar a adquirir conocimientos fáciles y convenientes con tecnologías informáticas (visualizadas, animadas, ricas en color y con sonido) se expresan cada vez más (Astakhova, 2016, p. 253). 
La disfunción cognoscitiva en el reconocimiento de la complejidad de las necesidades de las personas usuarias, conduce a una disminución en la asistencia de estas a las bibliotecas e, inclusive, en servicios de información en línea. Si bien el carácter excluyente de muchas tecnologías explica en parte esta disminución, son las barreras cognoscitivas las que determinan este fenómeno. Es así que las Ciencias cognitivas contribuyen en procesos de autocomprensión para el conjunto de profesionales en Bibliotecología.

\section{Conclusiones}

La primera aportación que se reconoce y expone en este trabajo, por parte de las tecnologías convergentes hacia la Bibliotecología, es de tipo conceptual. Tanto la Nanotecnología, la Biotecnología y las Ciencias cognitivas han reconfigurado en los últimos años su propia conceptuación de información. Esto ha hecho que profesionales de otras disciplinas, que tienen por objeto de estudio a la información, se detengan a re-pensar su naturaleza, dinámica y utilización.

Ahora bien, cada una de estas tecnologías convergentes realizan, de manera particular, aportaciones de orden práctico. Por ejemplo, los análisis teóricos que se realizan en la Nanotecnología han resultado en propuestas de factorización cuántica para la recuperación de información. La Biotecnología ha permitido que se adapten modelos de búsqueda y transmisión paralelas de bioinformación en métodos de organización y gestión de información documental. La aportación de las Ciencias cognitvas, por su parte, ha sido preponderantemente metodológica, en específico con la realización de estudios de comportamiento asociados con la búsqueda y uso de la información.

Huelgamencionarquelaexigenciasocialsobrelasaplicacionestecnocientíficas propugna para que estas cobren un carácter menos comercial y mucho más participativo en las vidas de las personas y de las sociedades. Además, el desarrollo responsable de las tecnologías convergentes debe contemplar la ética en la investigación, por lo que deben ser un apoyo para mejorar la calidad del trabajo en otras áreas, así como el desarrollo sustentable y el respeto general por la condición humana y otras formas de vida. La idea es que no solo los grupos de investigadores deben beneficiarse de estas tecnologías, sino, igualmente, el grueso de la población. La necesidad humana de comunicarse e informarse es generalizada. Si bien un investigador que desarrolla un órgano artificial debe usar la Nanotecnología -para comprender y construir el órgano a nivel celular-, la Biología -para comprender la función del órgano dentro del cuerpo- y las TIC para modelar y simular el órgano y sus funciones, también, la ciudadanía necesita beneficiarse de estas aplicaciones y comprender que también el gran público necesita información para participar y así tomar las mejores decisiones. 


\section{Referencias}

Astakhova, L. V. (2016). The information-and-cognitive activities of a person in a knowledge-based society. Scientific and Technical Information Processin, 43(4), 251-256.

Bellini-Leite, S. C. (2017). The revisionist strategy in cognitive science. En F. Adams, O. Pessoa y J. E. Kogler (Eds), Cognitive science: Recent advances and recurring problems (pp. 265-276). Delaware, Estados Unidos: Vernon.

Biro, J. C. (2011). Biological information: Definitions from a biological perspective. Information: Open Access Information Science Journal, 2(1), 117-139.

Chen, Z., Qiang, X. y Zhang, K. (2014). Molecular model for information addressing and transporting based on DNA nanotechnology. En L. Pan, G. Păun, M. J. Pérez-Jiménez y T. Song (Eds.), Bio-Inspired Computing: Theories and applications: 9th International Conference (BIC-TA 2014) (pp. 29-34). Heidelberg, Alemania: Springer.

Dalton-Brown, S. (2015). Nanotechnology and ethical governance in the European Union and China: Towards a global approach for science and technology. Cham, Suiza: Springer.

Dolničar, D., Boh-Podgornik, B., Sajovic, I., Šorgo, A. y Bartol, T. (2016). Assessing content and cognitive levels of information literacy in a group of life sciences university students. En S. Kurbanoğlu et al. (Eds.). Information Literacy: Key to an inclusive society: 4th European Conference (ECIL 2016) (pp. 403-411). Cham, Suiza: Springer.

Echeverría, J. (2003). La revolución tecnocientífica. Madrid, España: Fondo de Cultura Económica.

Floridi, L. (2011). The philosophy of information. Nueva York, Estados Unidos: Oxford University Press.

Fulbright, R. (2017). Cognitive augmentation metrics using representational information theory. En D. D. Schmorrow y C. M. Fidopiastis (Eds.). Augmented Cognition: Enhancing cognition and behavior in complex human environments: 11th International Conference (AC 2017), vol. II (pp. 36-55). Cham, Suiza: Springer.

Galindo, A. (2008). Del bit al qubit. En J. M. Díaz y F. Salto (Eds.), ¿Qué es información? Actas del I Encuentro Internacional de Expertos en Teorías de la Información: Un enfoque interdisciplinar (pp. 389-404). León, España: Instituto Nacional de Tecnologías de la Comunicación.

García, F. J. (1995). Paradigmas científicos en recuperación de la información. En F. J. García (Ed.), Organización del conocimiento en sistemas de información y documentación: Actas del I Encuentro de ISKO-España (pp. 99-112). Zaragoza, España: Universidad de Zaragoza. 
García, F. J. (2011). La pirámide de la información revisitada: Enriqueciendo el modelo desde la ciencia cognitiva. El Profesional de la Información, 20(1), 11-24.

Gordijn, B. (2008). Converging NBIC technologies for improving human performance: A critical assessment of the novelty and the prospects of the project. En B. Gordijn y R. Chadwick (Eds), Medical enhancement and posthumanity (pp. 225-235). Dordrecht, Países Bajos: Springer.

Guzmán, M. (2005). Ciencia de la información: Interdisciplinariedad y cambio de paradigma. Ciencias de la Información, 36(1), 3-11.

Hernández, P. (2009). Las ciencias cognitivas como base para explicar el fenómeno usuarios de la información. En J. Ríos (Coord.), XXVII Coloquio de Investigación Bibliotecológica y sobre la información: La investigación y la educación bibliotecológica en la sociedad del conocimiento. Coloquio realizado en el Centro Universitario de Investigaciones Bibliotecológicas, UNAM, Ciudad de México, México.

Hernández, P. (2013). El fenómeno usuarios de la información desde un enfoque cognitivo. Investigación Bibliotecológica: Archivonomía, Bibliotecología e Información, 27(61), 107-131.

Linares, J. E. (2008). Ética y mundo tecnológico. México, Fondo de Cultura Económica: UNAM, Facultad de Filosofía y Letras.

Lyre, H. (1996). Multiple quantization and the concept of information. International Journal of Theoretical Physics, 35(11), 2263-2269.

Middleton, L., Hall, H. y Raeside, R. (2019). Applications and applicability of social cognitive theory in information science research. Journal of Librarianship and Information Science, 51(4), 1-11.

Olivé, L. (2007). La ciencia y tecnología en la sociedad del conocimiento: Ética, política y epistemología. México: Fondo de Cultura Económica.

Paxton, A. y Tullett, A. (2019). Open science in data-intensive psychology and cognitive science. Policy Insights from the Behavioral and Brain Sciences, 6(1), 47-55.

Pedreño, A. (2009). Crisis económica, conocimiento y políticas para el desarrollo de la nanotecnología. Mundo Nano: Revista Interdisciplinaria en Nanociencias y Nanotecnología, 2(1), 78-87.

Recasens, F. (2018). Procesos de separación de biotecnología industrial. Barcelona, España: Universitat Politècnica de Catalunya, Iniciativa Digital Politècnica.

Roco, M. C. y Bainbridge, W. S. (Eds.) (2003). Converging technologies for improving human performance: Nanotechnology, biotechnology, information technology and cognitive science. Dordrecht, Países Bajos: Springer.

Rosenblueth, A., Wiener, N. y Bigelow, J. (1943). Behavior, purpose and teleology. Philosophy of Science, 10(1), 18-24. 
Savolainen, R. (2015). Cognitive barriers to information seeking: A conceptual analysis. Journal of Information Science Journal, 41(5), 613-623.

Shenton, A. K. (2009). Inferential information-seeking. Library Review, 58(5), 353-361.

Shunin, Y., Bellucci, S., Gruodis, A. y Lobanova-Shunina, T. (2018). Nonregular nanosystems: Theory and applications. Cham, Suiza: Springer.

Silva, A. R. da y Lima, G. A. B. de O. (2011). As categorias e a categorização cognitiva contemporânea: Enfoque sobre os olhares da biblioteconomia e ciência da informação e das ciências cognitivas. Revista Interamericana de Bibliotecología, 34(3), 301-312.

Sindelar, R. D. (2019). Dispensing biotechnology products: Handling, professional education, and product information. En D. J.A.Crommelin, R. D. Sindelar y B. Meibohm (Eds), Pharmaceutical biotechnology: Fundamentals and applications (pp. 239-251). Cham, Suiza: Springer.

Spink, A. y Cole, C. (2004). A human information behavior approach to a philosophy of information. Library Trends, 52(3), 616-628.

Stonier, T. (1996). La información y la estructura interna del universo: Una exploración en la física informacional. Madrid, España: Hacer.

Vega, G. (2009). Alfabetización informacional: Estudio sobre su apropiación en alumnos de primaria. (Tesis de doctorado). Facultad de Psicología, UNAM, Ciudad de México, México.

Venteño, M. G. (2013). La implementación de la nanotecnología en los procesos de gestión de la información en el gobierno (e-gobierno). En A. Cuadrado (Coord.), Gestión de información y conocimiento: Herramientas para el desarrollo local y comunitario (pp. 8-23). Extremadura, España: Fundación Ciencias de la Documentación.

Voutssás, J. (2011). La cadena de conservación en archivos digitales. En J. Ríos y C. A. Ramírez (Eds.), $7^{\circ}$ Seminario Hispano-Mexicano de Investigación Bibliotecológica y Documentación (pp. 307-328). México: UNAM, Centro Universitario de Investigaciones Bibliotecológicas.

Weisberg, Z. (2015). Biotechnology as end game: Ontological and ethical collapse in the "Biotech Century". NanoEthics: Studies of New and Emerging Technologies, 9(1), 39-54.

Weizsäcker, C. F. von (1949), The history of nature. Illinois, Estados Unidos: University of Chicago Press.

Wiener, N. (1988). Cibernética y sociedad. Buenos Aires, Argentina: Sudamericana.

Wiener, N. y Rosenblueth, A. (1946). The mathematical formulation of the problem of conduction of impulses in a network of connected excitable elements, specifically in cardiac muscle. Archivos del Instituto de Cardiología de México, 16(3), 205-265

Zibareva, I. V. (2015). A review of information resources on nanoscience, nanotechnology, and nanomaterials. Scientific and Technical Information Processing, 42(2), 93-111. 


\section{e-Ciencias de la Información}
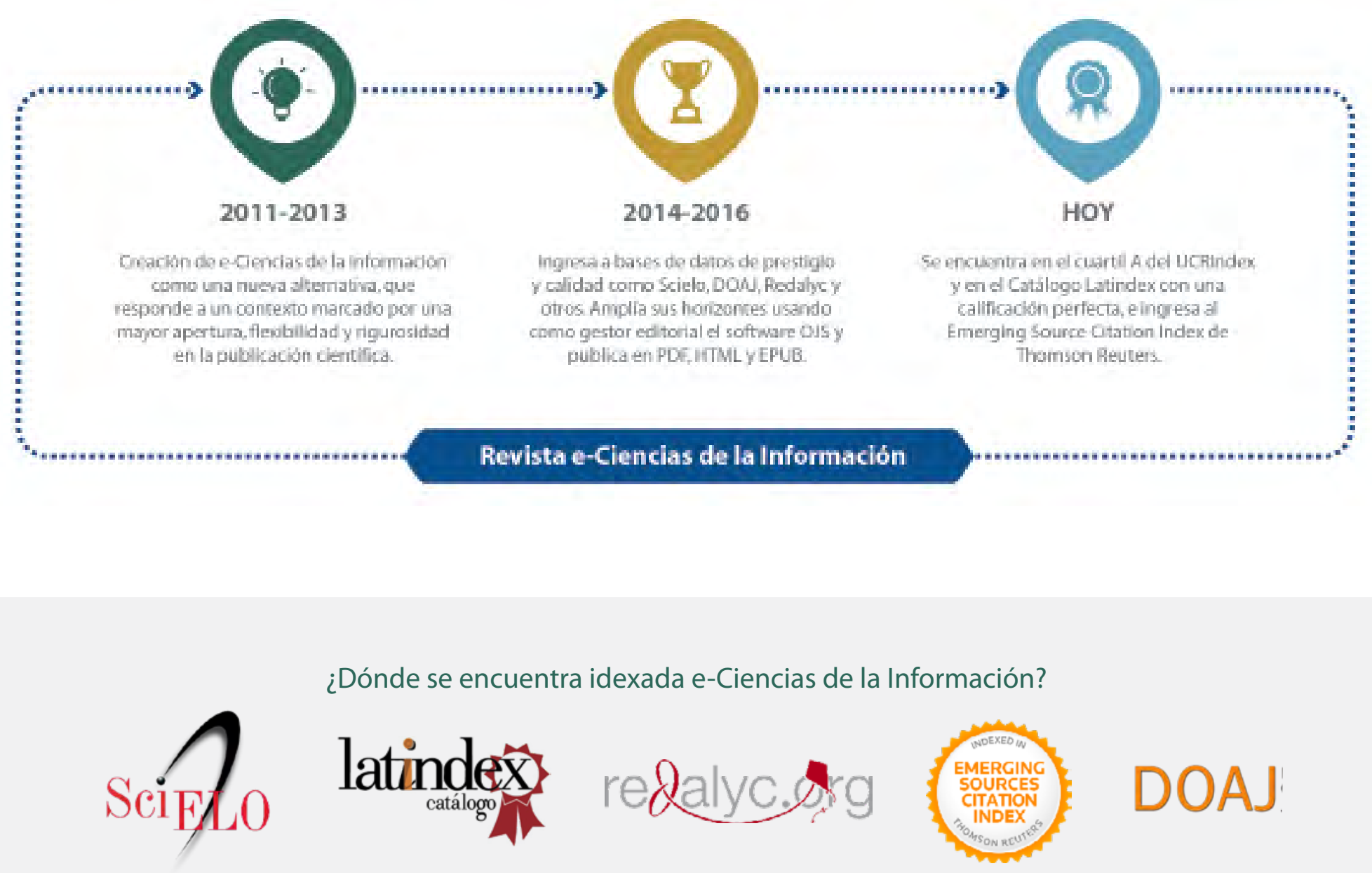

Para más información ingrese a nuestra lista completa de indexadores

¿Desea publicar su trabajo?

Ingrese aquí

O escríbanos a la siguiente dirección revista.ebci@ucr.ac.cr 